\title{
Alimentação, sociedade e cultura: temas contemporâneos
}

ELAINE DE AZEUEDO"

\section{Resumo}

Esta pesquisa teórico-conceitual coloca em evidência autores, temas e debates contemporâneos ligados à alimentação sob uma perspectiva sociopolítica e cultural. Ao levantar e analisar os diferentes estudos aqui mobilizados, foi possível definir cinco eixos temáticos da pesquisa sobre alimentação, cultura e sociedade. Tais temas exigem um diálogo interdisciplinar entre diferentes áreas, de forma a contribuir para a construção de uma perspectiva mais criativa e porosa sobre a alimentação, em sintonia com os debates sociais contemporâneos.

Palavras-chave: Alimentação. Sociedade. Cultura. Ativismo alimentar.

\section{Food, society and culture: contemporary issues}

\section{Abstract}

This theoretical and conceptual research highlights contemporary authors, issues and debates related to food through a political-cultural perspective. The analysis of the different studies here mobilized made possible to define five research topics involving food, culture and society. These issues require an interdisciplinary dialogue among different areas, in order to contribute to building a more creative and permeable perspective on food in line with the contemporary social debates. Keywords: Food. Society.Culture. Food Activism.

* Universidade Federal do Espírito Santo, Brasil 


\section{Introdução}

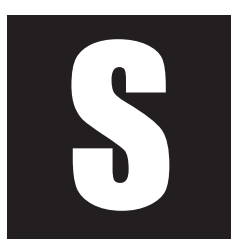

O comer é, assim, uma ação concreta de incorporação tanto de alimentos como de seus significados, permeada por trocas simbólicas, envolvendo uma infinidade de elementos e de associações capazes de expressar e consolidar a posição de um agente social em suas relações cotidianas (Carvalho; Luz, 2011, p.147).

digo capaz de gerar diferentes mensagens; potencial simbólico estrutural ou estruturante; construtora de identidades, gêneros e etnias; estratégia de prazer e lubrificação de interações sociais; fomentadora de intolerâncias e divisões sociais; mantenedora de vida ou promotora de problemas de saúde, controvérsias científicas, questionamentos éticos, angústias e riscos socioambientais, a alimentação configura-se como um objeto legítimo de análise social e de compreensão das premissas implícitas do viver em sociedade. As contribuições para esse campo de conhecimento caracterizam-se pela diversificação epistemológica, disciplinar, teórica e metodológica dos temas, pelo aumento de publicações acadêmicas ${ }^{1}$ e pelo crescente interesse de congressos científicos ${ }^{2}$, da mídia e de publicações generalistas ${ }^{3}$ dedicados à dimensão sociocultural da alimentação.

\footnotetext{
${ }^{1}$ Entre as publicações especializadas no tema da alimentação e cultura, destacam-se: Appetite; Food \& History; Food and Foodways; Gastronomica; Food, Culture and Society;

${ }^{2} \mathrm{O}$ encontro científico anual da International Sociology Association de 2016 propôs um grupo de trabalho que discute a alimentação e agricultura. Ver em: https://isaconf.confex.com/isaconf/forum2016/webprogrampreliminary/Symposium287.html Acesso em: 4 Mar 2016

${ }^{3}$ Destaque para duas publicações do início de 2015: a Revista de História da Biblioteca Nacional, de abril, cujo tema "Decifrando o ato de comer" discute diferentes sentidos da comida e a Revista Cult, de fevereiro, que apresenta o "Dossiê Gastronomia é Cultura? Formação, Identidade e História da Alimentação".
} 
Observa-se, também, o fortalecimento de instituições - como a FOST (Social \& Cultural Food Studies), o European Institute for the History and Culture of Food, a Agriculture, Food \& Human Values Society (AFHVS) e a Association for the Study of Food and Society (ASFS), fundadas em 2003, 2001, 1987 e 1985 respectivamente - que se ocupam de estudos interdisciplinares sobre alimentação, agricultura e sociedade e fomentam revistas e encontros científicos regulares envolvendo sociólogos, antropólogos, filósofos e outros pesquisadores das Ciências Humanas e Sociais. Apesar dessa recente efervescência, a alimentação foi, por muito tempo, uma temática social negligenciada. Tal condição pode ser explicada por seu vínculo a uma atividade doméstica, mundana e sem glamour, de domínio tradicional das mulheres, cuja produção remete ao meio rural, distanciado do apelo intelectual de teóricos masculinos atuando no meio urbano que dominaram historicamente as Ciências Sociais, especialmente no seu início (Beardsworth; Keil, 1997; Mintz, 2001).

Poulain (2004) mostra que, em um primeiro momento histórico, estendendo-se do início das Ciências Sociais até os anos 1960, alguns cientistas sociais debruçaram-se sobre o estudo da alimentação sob um contexto sensorial e comportamental, dissolvido na Antropologia e Sociologia da cultura. O fato alimentar foi analisado numa perspectiva histórica e mágica, como construtor de identidades sociais, "um lugar de indexação de outros fenômenos sociais" (op. cit. p. 152) em autores como Émile Durkheim, Mauricie Halbwachs, Chombart de Lauwe, Leroi-Gourhan, Radcliffe-Brown, Bronisław Malinowski, Edward E. Evans-Pritchar e Audrey Richards, pioneira da antropologia da alimentação.

Mintz (2001) afirma que, até os anos 1980, o estudo antropológico da comida, apesar de gradualmente crescente, ainda não era assumido como tema de relevância antropológica, o que só foi acontecer com o aparecimento de um mercado mundial de alimentos, a partir da 
década de 1990. Poulain (2004) e Beardsworth; Keil (1997) ressaltam outro momento anterior a esse, após os anos 1960, no qual a alimentação ganhou o status de objeto de estudo sociológico em autores franceses e anglo-saxões como Claude Lévi-Strauss, Mary Douglas, Allan Warde, Leo Moulin, Jean-Paul Aron, Igor de Garine, Claude e Christiane Grignon, Annie Hubert, Sidney W. Mintz, Marvin Harris, Jean-Pierre Poulain, Jean-Louis Lambert, Nicolas Herpin, Jean Pierre Corbeau, Jack Goody, Allan Beadsworth, Teresa Keil, Roland Barthes, Warren J. Belasco, Stephen Mennel, Anneke van Otterloo e Anne Murcott. Nesse período, a alimentação passa a ser percebida como construtora da identidade individual na perspectiva do cientista social francês Claude Fischler (1988), e serve de análise do comportamento e da diferenciação social para Pierre Bourdieu (1984). Cabe destacar, ainda, que a tese de Claude Fischler sobre o onívoro, publicada originalmente em 1990, se coloca como um trabalho de liderança na área de Sociologia da Alimentação. Nessa mesma época, Jean-Louis Flandrin e Massimo Montanari (1998) apresentaram uma coletânea histórica sobre alimentação e a evolução da culinária e dos hábitos alimentares, a partir da pré-história, que contribuiu significativamente para a compreensão de práticas sociais e agrícolas, e de crenças religiosas.

No contexto brasileiro, é possível incluir os relatos históricos de Pero Vaz de Caminha, de Jean de Léry, de André Thevet e de Hans Staden sobre os hábitos alimentares dos indígenas locais, compilados por Rial (2005). Também merece menção a obra de Carlos Rodrigues Brandão, Plantar, colher e comer, publicada em 1981, que se debruça sobre a vida cotidiana do campesinato goiano por meio da alimentação.

Klaas e Ellen Woortmann foram precursores dos estudos sobre alimentação em grupos tradicionais (junto com Otávio Velho). O casal de antropólogos elaborou o Relatório sobre Hábitos e Práticas Alimentares, com foco para a relação entre alimentação e gênero, família e parentes- 
co, bem como percepções sobre impactos da produção de alimentos no meio ambiente. Klaas Woortmann foi também responsável pela realização de um dos primeiros estudos qualitativos da produção de alimentos em Sergipe, a convite do Instituto Nacional de Alimentação e Nutrição, em 1978 (Schwade; Woortmann, 2014).

Destacam-se os brasileiros Gilberto Freyre (1977) cuja abordagem de alimentação inclui uma miríade de aspectos sociais, e foi resumida por Silva (2014), e por Câmara Cascudo. Este último publicou, em 1967, a primeira edição de História da Alimentação no Brasil (Cascudo, 2014). Por fim, alude-se ao ilustre Josué de Castro, precursor da denúncia da fome como expressão biológica de inequidades sociais no Brasil, na obra Geografia da Fome, de 1947. Mais recentemente, Maria do Carmo Soares de Freitas (2003) aborda o mesmo drama no Brasil contemporâneo, compactuando com Arnold (1988, p. 3), que vê a alimentação como "poder na forma mais básica, tangível e inescapável".

A partir do ano 2000, o tema, percebido pelos editores da revista Food and Foodways ${ }^{4}$ como um fenômeno social penetrante que demanda muitas disciplinas para abarcar sua complexidade, ganha cada vez mais atenção e aparece endossado por autores contemporâneos das Ciências Sociais e Políticas, da Psicologia Social, da Economia, da História, da Saúde, da Gastronomia, do Direito, da Geografia, da Nutrição, do Meio Ambiente, do Jornalismo e dos estudos de sistemas agroalimentares, de gênero e sexualidade, de ética e de consumo.

Diante dessa pluralidade de áreas, torna-se um desafio apontar os autores contemporâneos de maior destaque na área de alimentação, sociedade e cultura, mas mesmo sabendo do risco de omitir nomes de relevância mencionam-se os trabalhos de Peter Atkins e lan Bowler (2001),

${ }^{4}$ Citação disponível na página eletrônica da Revista Food and Foodways, http://www.researchgate.net/journal/0740-9710_Food_and_Foodways Acesso em: 5 fev.2015. 
Marion Nestle (2003; 2002), George Ritzer (2004), Eric Schlosser (2012) e John Coveney (2006), Michel Pollan (2007; 2008), John Germov e Lauren Williams (2008), Paul Rozin (2008), Tim Lang, David Barling e Martin Caraher (2009), Michael Carolan (2012), Carole Counihan e Penny Van Esterik (2012), Jean-Pierre Poulain (2004; 2012), Jonh Coveney (2006) que publica junto com Paul Ward e Julie Henderson (2010) e David Inglis e Debra Gimlin (2009). Importante apontar o trabalho de Jesús Contreras e Mabel Garcia Anaíz (2011) que, junto com outros pesquisadores de diferentes áreas como Miriam Bertand Vilá e Antoni Riera Melis, fazem parte do observatório de investigação alimentar, Observatorio de la Alimentación (ODELA), vinculado à Universidade de Barcelona. Harry West e Jakob Klein, entre outros pesquisadores, fazem parte do SOAS Food Studies Centre, da Universidade de Londres, outro centro interdisciplinar dedicado ao estudo das dimensões políticas, econômicas e culturais de alimentos, da produção ao consumo.

No Brasil, ganham destaque trabalhos do sociólogo Carlos Alberto Dória $(2015 ;$ 2014), a abordagem de identidade cultural e alimentação para a qual se dedica a antropóloga Maria Eunice Maciel (2005) da Universidade Federal do Rio Grande do Sul, além da análise psicossocial da alimentação em Angelina Bulcão Nascimento (2007) e a reflexão sobre o corpo, o comer e a comida sob as premissas das tradições alimentares soteropolitanas em Lígia Amparo da Silva Santos (2008), da Federal da Bahia.

A antropóloga Janine Collaço coordena o grupo Consumo, Cultura e Alimentação (CNPq/ Universidade Federal de Goiás) que tem como foco a questão do consumo e as influências de lógicas culturais na seleção e escolha alimentares. Collaço (2013) desenvolveu sua tese sobre a cozinha italiana e construção identitária em São Paulo. No mesmo grupo, com especial interesse no ativismo alimentar, estão os sociólogos Elaine de Azevedo, da Universidade Federal do Espírito Santo, e Daniel Coelho 
de Oliveira, da Universidade Estadual de Montes Claros, que também faz parte do Grupo de Estudos de Consumo vinculado ao Programa de Pós-Graduação de Antropologia da Universidade Federal Fluminense. Esse grupo é composto de outros pesquisadores dedicados a discussão de tendências e ideologias do consumo alimentar como Lívia Barbosa (Universidade Federal Fluminense), Fátima Portilho (Universidade Federal Rural do Rio de Janeiro), entre outros.

Evidenciam-se, também, os autores das áreas de Antropologia da Alimentação e da Nutrição Social como Renata Menasche, Rosa Wanda Diez Garcia, Denise Cavalcanti de Barros, Denise Oliveira e Silva, Miriam Ribeiro Baião, Maria Claudia da Veiga Soares Carvalho e Shirley Donizeti Prado, entre outras, que participam dos seguintes grupos acadêmicos de pesquisa: o Núcleo de Estudos e Pesquisas em Alimentação e Cultura da Escola de Nutrição da Universidade Federal da Bahia (ENUFBA); o Núcleo de Estudos Interdisciplinares Alimentação e Cultura da Universidade Federal do Rio Grande do Sul (UFRGS) e o Grupo de Estudos sobre Cultura e Alimentação (Nectar) da Universidade Estadual do Rio de Janeiro. Inúmeros pesquisadores estão vinculados a duas redes importantes: a Rede Interinstitucional de Alimentação e Cultura, vinculada à Escola Nacional de Saúde Pública Sergio Arouca, da Fundação Osvaldo Cruz (FIOCRUZ), e a Naus, Rede Ibero Americana de Pesquisa Qualitativa em Alimentação e Sociedade, que tem como principal objetivo fortalecer pesquisa qualitativa na área sociocultural da alimentação. Importante destacar o lançamento, em 2016, do Observatório Brasileiro de Hábitos Alimentares (OBHA), um projeto desenvolvido por pesquisadores da Fundação Oswaldo Cruz, de Brasília, em parceria com diversas instituições nacionais e internacionais, que objetiva promover e divulgar pesquisas e material acadêmico e informativo, assim como eventos sobre alimentação e hábitos alimentares realizados no Brasil. 
Esse ensaio teórico conceitual e reflexivo não tem a pretensão de esgotar tão ampla temática e se propõe centralmente a ampliar o espaço de discussão. O estudo mobiliza autores dedicados ao estudo da Alimentação, Sociedade e Cultura, de forma a ressaltar algumas das suas orientações de pesquisas e eixos temáticos já explorados e a fomentar novos estudos. Assim, o artigo discute tais estudos a partir da elaboração de cinco eixos temáticos apresentados a seguir.

\section{Temáticas contemporâneas} da área de Alimentação, Sociedade e Cultura

Ao levantar e analisar os diferentes estudos aqui mobilizados, foi possível definir pelo menos cinco eixos temáticos da pesquisa sobre alimentação, cultura e sociedade: (1) a discussão que envolve riscos, controvérsias e a ampla abordagem da (in)segurança alimentar e nutricional; (2) o alimento como tema frutífero para explorar os múltiplos significados da globalização e da urbanização uma vez que expõe a complexidade desses fenômenos e como os mesmos afetam a seleção dos alimentos; (3) o eixo chamado aqui "Comer de outras formas" que abriga discussões sobre alimentação e ética, gênero, sexualidade, literatura, cinema e artes; (4) as abordagens sobre a gastronomia, o papel do chef e suas implicações culturais e políticas; (5) o ativismo alimentar que coloca em evidência diversos movimentos transformados em repertórios de ações coletivas: Agroecologia, Freeganismo, Locavorismo, Agricultura Orgânica, Vegetarianismo, entre outros.

Ressalta-se que, de toda forma, tal recorte torna-se arbitrário e autoral. É uma possível perspectiva de análise e, por isso, incorre na possibilidade de não contemplar todas as temáticas de tão amplo escopo. O primeiro eixo se dedica à problemática dos riscos e ansiedades produzi- 
dos pelo sistema agroalimentar moderno e as consequentes controvérsias que circundam o comer contemporâneo.

\section{Riscos, Controvérsias e (in)segurança alimentar e nutricional}

A produção de alimentos envolve complexas interações humanas e naturais que foram se complexificando no decorrer do desenvolvimento da humanidade e acabaram por expor imbricadas relações de poder e inequidades, bem como repercussões ambientais, sociais e sobre a saúde humana, que se tornaram objeto de clamor de diferentes movimentos sociais e ambientais.

Desde o alerta 'gastro-anômico' de Claude Fischler (1995) e sua discussão de cacofonia alimentar, a ansiedade relacionada às incertezas e controvérsias em torno do tripé alimentação-saúde-doença tem se intensificado ultimamente - ou pelo menos tem se mostrado de forma mais expressiva. Um volume enorme de notícias sobre o tema da alimentação passou a ganhar espaço na grande mídia e na internet, a que tem acesso o público leigo da sociedade globalizada, na qual a circulação de informação é uma de suas características centrais. Assim, a frágil fronteira entre a categoria de alimento saudável e a de alimentos que apresentam riscos, e as consequentes controvérsias científicas geradas por tal perspectiva exploradas em Azevedo (2013a, 2011,), David e Guivant (2012) e Guivant (2006) são questões fundamentais acolhidas pelas Sociologias da Alimentação e do Conhecimento Científico.

O estudo das controvérsias permite encaixar a ciência em um contexto de atividade humana como outra qualquer que envolve jogos de poder, erros, fragilidades e impotências. Em se tratando da ciência da Nutrição, a relação controvérsias e alimentação torna-se pertinente para compreender as limitações da ciência, para dar visibilidade aos confron- 
tos entre especialistas e entre leigos e peritos, e para evidenciar os dissensos e riscos que permeiam o que é um alimento saudável, trazendo à tona a pluralização e a democratização dos saberes que definem tal conceito.

A temática da confiança impulsionada por Luhmann (1979) nas Ciências Sociais dialoga com a dos riscos alimentares e das controvérsias científicas e pode ser encontrada em estudos de Truninger (2013), Allain e Guivant (2010). No contexto de individualização dos riscos ligados à alimentação, Ward e colaboradores (2010) remetem a Zivokic e colaboradores, cujo estudo mostra as implicações de um debate moral e legal que delega, com apoio da mídia, aos pais, especialmente à mãe a responsabilidade pela obesidade infantil. No Brasil, o Ministério da Saúde tem se dedicado a alertar as diversas mudanças políticas, econômicas, sociais e culturais que evidenciaram transformações no modo de vida da população e suas graves repercussões sobre a obesidade e outras doenças não-transmissíveis ${ }^{5}$, assim como a influência da propaganda sobre a obesidade infantil.

Para além dos riscos alimentares contemporâneos, permanece a veIha chaga que fomentou os primeiros estudos sociológicos no país. A problemática da fome e da desnutrição foram substituídas pela complexa discussão da (in)segurança alimentar e nutricional, assumida no Brasil pelo Conselho de Segurança Alimentar e Nutricional (CONSEA) (Brasil, 2007a), sob grandes desafios como o combate ao elitismo alimentar, a bulimia, a anorexia, a obesidade e as doenças não-transmissíveis causadas pela dieta desequilibrada; a luta pelo acesso igualitário à água e ao alimento saudável como direito humano, em detrimento da condição de mercadoria ou privilégio; a promoção da soberania alimentar e nutricional; a luta contra o desperdício de alimentos; o direito do consumidor/comedor e a qualidade dos alimentos; o conflito entre a conveniência e a qualidade alimen-

\footnotetext{
${ }^{5}$ Guia disponível em: http://portalsaude.saude.gov.br/images/pdf/2014/novembro/05/Guia-
} -Alimentar-para-a-pop-brasiliera-Miolo-PDF-Internet.pdf Acesso em: 4 Jan 2015. 
tar dos produtos industrializados; o fomento à agroecologia e aos sistemas agroalimentares sustentáveis; a alimentação redefinindo fronteiras entre os meios urbano e rural; a alimentação como construtora de simbolismo, como bem cultural e como estratégia de socialização, premissas corrompidas durante o processo de urbanização moderno. Seria impossível listar aqui a diversidade de estudos e autores dedicados à construção de uma referendada política de Segurança Alimentar e Nutricional que colocou o Brasil como referência internacional no combate à fome e erradicação da pobreza. Nesse âmbito, releva-se pertinente o enfoque do impacto do sistema jurídico sobre as agrobiodiversidade e o direito dos agricultores explorado por Santilli (2009).

As políticas públicas de Alimentação e Nutrição e de Segurança Alimentar e Nutricional e suas formas específicas de organização, de promoção a participação social, e de protagonismo enquanto atores coletivos e movimentos sociais, estão associadas a processos históricos de organização política da saúde que remetem ao Movimento de Reforma Sanitária Brasileiro da década de 1970 e ainda permanecem distantes dos estudos empíricos de Sociologia e Ciência Política contemporâneas. Da mesma forma, a violação do direito humano a uma alimentação adequada e saudável para determinados grupos discriminados - negros, indígenas, população de rua, população do meio rural, sem terras, imigrantes - bem como a apropriação e a privatização de recursos genéticos alimentares, e a subordinação da pesquisa e do desenvolvimento tecnológico alimentar aos imperativos do mercado também merecem ser contempladas pelo olhar das Ciências Sociais, que vêm sofrendo forte influência dos processos de mundialização e globalização que serão discutidos a seguir. 


\section{Globalização, urbanização e seleção dos alimentos}

O alimento é um tema frutífero para explorar os múltiplos significados da globalização, uma vez que expõe a complexidade de um peculiar fenômeno que transcende o aspecto econômico. Apesar das suas claras relações com a cultura local, a religião, o gosto, a tradição, o simbolismo e a identidade, a comida tem sido produzida como uma mercadoria sob as premissas de um sistema e de uma política agroalimentar de caráter global, dominada por corporações agroalimentares transnacionais, o que envolve uma forma legitimada de agrobiopoder e de ameaça à soberania alimentar, além de impactos culturais e socioambientais significativos. Desse modo, a globalização pode ser compreendida sob a ótica da alimentação e sofre também influência da mobilidade das pessoas e suas ideias sobre culinária, gosto e nutrição (Grew, 2011). Dessa forma, o estudo de Paul Rozin (1976) sobre os aspectos psicobiológicos que interferem nas escolhas alimentares, a neofobia e neofilia e a manutenção de gostos e preferências é ainda relevante na análise dos processos de intensificação migratória e urbana.

Outra estratégia para compreender a seleção dos alimentos pode ser encontrada em Beardsworth e Keil (1997), que criaram o conceito de menu. Os sociólogos definiram três tipos de menus que guiam as escolhas alimentares: os menus tradicionais, que apoiam crenças e proibições; os menus morais, que selecionam a comida a partir de critérios étnicos, políticos, ecológicos, ambientais e éticos; e os menus racionais, baseados no modelo de racionalização da dieta, que promovem a escolha alimentar a partir de critérios científicos para perda peso e aumento da performance física ou mental. Esses menus racionais se subdividem em menus de conveniência, cujo objetivo é minimizar o tempo dedicado ao ato de alimentar-se; os menus econômicos, que tem no custo o delineador das 
escolhas alimentares, e os menus hedonísticos que tem como foco da escolha o prazer gustatório.

A perspectiva ideológica do Nutricionismo ${ }^{6}$ que reduz a comida a seus componentes bioquímicos dialoga com essa ideia de racionalização da dieta, promovendo uma alimentação individualizada, desprovida de valores culturais e funções sociais, endossa o fenômeno da medicalização da Nutrição, impulsionado pela ideia de saudabilidade do corpo magro. Para além de diferentes repercussões sobre a saúde advindas desse modelo interventivo, o qual contribui para a construção da chamada "lightização da existência" sob uma "nova ordem corporal" (Santos, 2008, p.18), presume-se que a tendência do aumento da prescrição e do consumo de alimentos industrializados de caráter dietoterápicos (light, diet) e de cápsulas sintéticas que substituem a comida carece de estudos que analisem seu impacto social. Teme-se que o fortalecimento da indústria de suplementos, por exemplo, voltados para um mercado solvível e rentável e para o tratamento de disfunções que impactam os grupos mais afluentes da sociedade, promova a elitização da Nutrição e a consequente redução de interesse e de intervenções para promover segurança alimentar e nutricional. Essa mesma lógica pode ser aplicada para analisar o estímulo às tecnologias agroalimentares - como a transgenia e a nanotecnologia aplicadas à produção de comida - em detrimento da lógica de fomento da segurança e soberania alimentar e dos sistemas agroalimentares sustentáveis.

O universo do consumo de alimentos como construtor de identidades individuais e coletivas, do consumo sustentável e do consumerismo político, analisados em Micheletti (2003) e Spaargaren (2005), estimulou estudos como o de Portilho e colaboradores (2011), que buscam reconhecer o perfil e as estratégias do consumidor cidadão ao fazer escolhas alimentares a partir de premissas socioambientais. A urbanização também

${ }^{6}$ Termo criado por Gyorgy Scrinis (2008). e popularizado pelo jornalista Michel Pollan. 
influenciou os estudos da alimentação. O processo descrito por Verthein e Vázques-Medina (2015) como desritualização da comida evidencia-se com a flexibilização de horários, locais e ritmos alimentares das cidades (mas não restritos ao meio urbano).

Flammang (2009), em seu livro Taste of civilization, analisa os rituais das refeições e de preparação de alimentos como bases para uma educação voltada à promoção da civilidade, ao cultivo da democracia e ao exercício da cidadania. A crise de civilidade que a autora percebe nos Estados Unidos é analisada sob a ótica da falta de tempo dedicada à arte do diálogo aprendida à mesa, no momento de compartilhar refeições. Para a autora, reflexão e generosidade são sentimentos implícitos à comensalidade. Sob o mesmo ideal de promoção da comensalidade, o estudo de Woolley e Fishbach (2017) examina as consequências do consumo de alimentos incidental como forma de estimular confiança e cooperação entre estranhos. A pesquisa mostra que o consumo de alimentos influencia a resolução de conflitos e promove cooperação. As autoras defendem que pessoas que seguem o mesmo tipo de dieta tendem a entrar mais rapidamente em concordância em alguns tipos de discussões e processos de decisão. Esses estudos devem contribuir para a análise da temática que envolve comida e migração. As chamadas migratory meals têm como meta aproximar, através de refeições compartilhadas, os nativos de diferentes países dos imigrantes e refugiados. Os estudiosos se preocupam em investigar os festivais e encontros gastronômicos e restaurantes de novos imigrantes e refugiados transnacionais e o seu papel em promover acoIhimento e inserção sociais, diminuição do preconceito e construção da identidade desses atores globais como fez Fedora Gasparetti (2009) em sua análise da alimentação trazida pelos imigrantes senegaleses na Itália.

Na outra ponta da mesa, há quem questione essa 'overdose social', termo usado pela designer holandesa Marina Van Goor, que criou a rede 
de restaurantes europeia Eenmaal especializada em servir refeições para uma pessoa. O apelo dessa rede é que não é preciso estar conectado o tempo todo, e não há nada de mal em comer sozinho. Além disso, na percepção de Emmy Ronken, chef de um dos restaurantes da rede em Amsterdã, essa experiência implica em um estado mais refinado de atenção e meditação voltado para os diferentes sentidos que envolvem o comer, o que pode, para ela, ser considerado uma nova experiência social (Moskovics, 2015).

Reverberam, nessa linha, os estudos sobre o novo status da comida de rua, o comer fora de casa, o comer trabalhando. Verthein e Vázques-Medina (2015) fazem uma alusão a Ascher (2005) e seu conceito de hipermodernidade alimentar, uma reflexão acerca da liberdade do comedor eclético hipermoderno frente ao controle da indústria alimentar, a culpa de comer o que deseja, e as restrições impostas por suas escolhas, na forma de distúrbios como a obesidade e a anorexia. Diante da inescapável condição contemporânea reflexiva abordada por Giddens (1991), que afeta todas as escolhas cotidianas, é natural que os modos de comer contemporâneos se pluralizem e sejam analisados sob novas lentes. Novas formas de comer.

\section{Comer de outras formas}

A chamada para um simpósio interdisciplinar sobre Alimentação e Cultura, realizado no início do ano de 2015, na Universidade de Lancaster, Inglaterra, sob o tema "Eating Otherwise" 7 , algo como "Comer de outra forma", definiu bem a amplitude das relações possíveis de se estabelecer

\footnotetext{
${ }^{7}$ Chamada do Interdisciplinary Symposium on Food and Culture, realizado em 28 fev a 1 o. mar de 2015 na Universidade de Lancaster, Inglaterra. disponível em:

https://eatingotherwise.files.wordpress.com/2014/08/eating-otherwise-cfp001.pdf. Acesso em: 26 Mar 2015.
} 
com a comida. As áreas de investigação do encontro incluíram: alimentação, literatura, cinema, TV e artes; alimentação e biopolítica; alimentação e tecnologia; alimentação e corpo; alimentação na filosofia; comida, lugares e memórias; alimentação e religião e movimentos alimentares canalizando para a produção de estudos de alimentos e animais; alimentos e sexo (comidas afrodisíacas, comida na pornografia, porn foods); comidas profanas e sagradas; comidas sinistras, subversivas e góticas (entomofagia, placentofagia, coprofagia e necrofagia); canibalismo; alimentação do passado, presente e futuro; alimentação pós-humana; alimentação, gênero e Teoria Queer; desordens alimentares e alimentação saudável; alimentação, prazer e culpa. O texto de introdução desse encontro provoca uma série de possibilidades que o estudo da comida abre:

(...) pode-se comer a própria comida, mas pode-se também falar sobre ela, ou até mesmo falar com ela ${ }^{8}$; pensar sobre a comida ou através dela; lembrar-se através dela; sentir e reviver sensações através dela; estimular-se sexualmente através da comida; experimentar a si mesmo e ao mundo através da comida; tentar efetivar mudanças através da alimentação, como na greve de fome; e, é claro, pode-se usá-la para a tortura, como no caso de uma alimentação forçada ${ }^{9}$.

O questionamento do antropocentrismo que motivou discussões sobre o direito dos animais em Singer (2004) e a visão feminista-vegana de Carol Adams (1990; 2003), em suas publicações The Sexual Politics of Meat e The Pornography of Meat, mobilizaram autores como Rudy (2012) e a

\footnotetext{
${ }^{8} \mathrm{O}$ falar com a comida remete ao fenômeno dos adolescentes chineses que adotam, conversam e passeiam com acelgas e repolhos para driblar a solidão das grandes cidades e sentir-se da mesma forma que um vegetal; tais casos veiculados na mídia sensacionalista da internet certamente provocam, se não ainda a imaginação sociológica, a perplexidade e curiosidade que movem a humanidade. Disponível em: http://www.megacurioso.com.br/oriente-bizarro/43093-chineses-solitarios-driblam-a-falta-de-amigos-com-repolhos-de-estimacao. htm. Acesso em: 2 Mar 2016.

${ }^{9}$ Texto introdutório do Interdisciplinary Symposium on Food and Culture supracitado, disponível em: https://eatingotherwise.files.wordpress.com/2014/08/eating-otherwise-cfp001.pdf Acesso em: 26 Mar 2015.
} 
ecofilósofa Val Plumwood (2000) que exploram abordagens sobre vegetarianismo, locavorismo, ética e feminismo, revelando uma abordagem de gênero no prato. Na mesma direção, Simonsen (2012) lança um 'manifesto Queer vegano' para discutir o potencial do veganismo de romper o vínculo 'natural' entre as formações de gênero e o consumo de produtos animais.

Klaas Woortmann (1986) já afirmava que o gênero também se constrói no plano das representações, através da percepção da comida. Siliprandi (2004) pesquisa a insegurança alimentar e as relações de gênero, e Dória (2012) explora a desfeminilização da cozinha, entre outras temáticas acolhidas no Dossiê Gênero e Alimentação, coordenado por Algranti e Asfora (2012).

Para além da análise antropológica de alimentos com propriedades afrodisíacas em culturas tradicionais, ainda se produzem abordagens interessantes sobre a relação entre comida e sexo. Ambos são fenômenos biológicos e culturais, prazerosos, nutritivos e permitem explorar variedades de receitas. A sedução da comida permite usá-la como metáfora sexual implícita nas ideias de 'comer' para manter relações sexuais (Woortmann, 2004); de referências alimentares para enfatizar o erotismo e as desigualdades de gênero como na expressão 'homem/ mulher gostoso/a' ou no fenômeno das mulheres frutas ${ }^{10}$. Para Nascimento (2007, p. 111), o

vocabulário amoroso se entrelaça constantemente com o gastronômico [...] implícito nas expressões "pele de pêssego", "olhos amendoados" ou "cor de avelã", "boca de cereja", "lábios polpudos", "formas apetitosas", sem esquecer a "lua-de-mel" que, tantas vezes, acaba com o "caldo entornado".

A alimentação é mobilizada no cinema ${ }^{11}$, na literatura e nas artes visuais como personagem, inspiração, métodos para revelar personagens

\footnotetext{
${ }^{10}$ As mulheres frutas - jacas, peras, melancias, melão, moranguinhos - fenômeno de consumo do funk carioca e da mídia erótica.

${ }^{11}$ Ver Food and films Festivals: http://www.nycfoodfilmfestival.com/ Acesso em: 5 Fev 2015. Para mais informações sobre o tema comida e visualidades consultar: Azevedo e Peled (2016).
} 
ou agente relacional de tramas e emoções cotidianas. Práticas artísticas participativas, críticas e relacionais que incorporam o ativismo alimentar inspirado por questões políticas ou socioambientais são exploradas em estudo de Azevedo e Peled (2015), ambos pesquisadores do Grupo Diálogos entre Sociologia e Arte (DISSOA) da Universidade Federal do Espírito Santo. Já o Grupo Universalidades, Literatura e Alimentação (G.U.L.A) da Universidade Federal de Campina Grande, na Paraíba, destaca-se por seu "interesse de pensar a porção cultural e social da alimentação humana pela via da literatura, compreendendo-a como objeto de conhecimento [...]. O prazer da comida e o prazer do texto, como sugere Roland Barthes, reunidos para produzir um novo prisma para se enxergar a Nutrição"12.

A gastronomia também se difunde em programas de televisão, filmes e diferentes redes sociais como Instagram, Pinterest, Twitter ou Facebook. O site foodporn.com, entre tantos outros, é dedicado às experiências hedonistas da comida e da bebida. E há estudos específicos que se preocupam com a arte de 'regular o estômago', a gastronomia.

\section{O universo da gastronomia}

Dória (2015) enfatiza as mudanças que a gastronomia e o papel dos chefs vêm sofrendo e reconhece uma tripla dimensão da alimentação, que abarca a culinária (ou os processos físico-químicos de preparo dos alimentos), a gastronomia (ou o tratamento de um produto alimentar dentro de um determinado grupo social) e a gastronomização (ou a composição do signo alimentar expresso em domínios de marketing e publicidade).

Ainda no âmbito da gastronomia, há espaço para estudar e compreender o fenômeno dos foodies, termo criado em 1984 pelos escritores

\footnotetext{
${ }^{12}$ Informação disponível no site do G.U.L.A em: https://grupogula.wordpress.com/sobre/. Disponível em: 6 Fev 2015.
} 
ingleses Paul Levy e Ann Barr para designar pessoas, em especial homens, de alto nível intelectual, que têm um agudo interesse por experiências gastronômicas refinadas e gourmets. A proliferação dos chefs celebridades, dos programas de televisão e do chamado turismo enogastronômico-cultural pode ilustrar essa tendência ou "hobby de gente refinada" (Nascimento, 2007, p. 220). Eve Turow (2015) volta-se para a geração Yum, formada por jovens estadunidenses obsessivos por comida e por receitas e experiências gastronômicas, sentimento causado pela privação sensorial que a era digital promove. A comida e o comer fomentariam a emoção e os múltiplos sentidos que faltam aos solitários 'gastronautas' em frente das telas.

Por fim, as discussões sobre gastronomia responsável e ecogastronomia - do "prato ao planeta"13 - e a gastronomia regional aproximam o estudo da alimentação de uma dimensão identificada por Poulain (2004, p. 32) como "local de resistência identitária". Esse local se torna mais tangível em alguns estudos que se debruçam sobre o meio rural, onde se produz a alimentação e a história dos bastidores da comida. É o resgate de um espaço que abriga agricultores familiares locais vivendo sob uma natureza pluridimensional e que pode produzir um senso de pertencimento, solidariedade e comunidade - ou a quebra desse sentido. Tal discussão é assumida por Azevedo $(2015 ;$ 2012) ao abordar o movimento dos locávoros, da Agroecologia e dos sistemas agroalimentares sustentáveis sob a ótica do ativismo alimentar.

\footnotetext{
${ }^{13} \mathrm{O}$ Slow Food assume a proposta da ecogastronomia relacionando o prazer de comer com a consciência e a responsabilidade ambiental, estabelecendo conexões entre "o prato e o planeta". Citação disponível em: http://www.slowfoodbrasil.com Acesso em: 15 out 2014.
} 


\section{O ativismo alimentar como desdobramento do ativismo político}

O ativismo alimentar é considerado por Rudy (2012) como um dos movimentos sociais mais vibrantes da atualidade, que se debruça sobre questões que vão além da comida e que complexificam a relação do ser humano com os outros reinos da natureza.

O ativismo alimentar apresenta-se como uma vertente do ativismo político que emergiu na década de 1960, como uma perspectiva mais porosa, comprometida e criativa de fazer política. Presente historicamente em todo tipo de sistema político, o ativismo político, recorrentemente associado a causas progressistas e à promoção da equidade e dos direitos das minorias, privilegia a militância ou ações contínuas, inovadoras e energéticas, que objetivam uma efetiva transformação da realidade, a partir de estratégias coletivas. O ativismo político envolve processos participativos e diferentes formas de comportamentos coletivos que incluem a defesa, a propagação e a manifestação pública de ideias; o boicote no ato de consumo (buycott); a realização de manifestações públicas organizadas (protestos, comícios, marchas, recrutamento de simpatizantes); a prospecção porta-a-porta; o fomento a diferentes tipos de campanhas para levar as visões da sociedade civil local e/ou global para âmbitos políticos internacionais; e o apoio a manifestos favoráveis a uma determinada causa ou contra algo que prejudique a mesma (Martin, 2007; Norris, 2007).

Tal movimento social, no seu surgimento, privilegiou as ruas e os emergentes meios de comunicação em massa para questionar as diferentes formas de autoridade, os valores e as instituições do establishment e divulgar protestos antiguerras, a favor da liberdade de expressão e de apoio a movimentos ambientais e sociais das minorias étnicas, raciais e sexuais. Mais recentemente, conta com a sofisticação das tecnologias de informação e comunicação, especialmente a internet, o que contribui para uma 
renovação das formas e locais de organização de tais movimentos e para a constituição e a existência de novos entes políticos (Machado, 2007).

Para Noris (2007, p. 639), esse processo evolutivo do ativismo político assistiu ao declínio da autonomia dos Estados-nação e complexificou as "ações orientadas por cidadãos/ citizen-oriented actions", aquelas relacionadas especialmente a eleições e partidos, sob as premissas dos "repertórios orientados por uma causa/ cause-oriented repertories" que se voltam para assuntos específicos e preocupações políticas de consumidores politizados. $\mathrm{O}$ ativismo político assume, cada vez mais, a tomada de diferentes espaços - virtuais, comunitários, públicos, privados, sem fins lucrativos, cotidianos e íntimos - legitimando-os como cabines eleitorais e campos de ação política e, assim sendo, tornou-se mais difícil para os cidadãos utilizarem o processo eleitoral, os partidos nacionais, o parlamento e os legislativos nacionais, como única forma de construção de políticas públicas desafiadoras, reforçando a necessidade de repertórios alternativos e horizontalizados para fomentar a mobilização e a expressão política.

Valores utópicos modernistas, principalmente aqueles que padronizavam tendências sob uma perspectiva universal ocidental, vêm sendo questionados, e pode-se dizer que os espaços das grandes projeções futuristas aliados à racionalização dos espaços públicos estão sendo gradual e paralelamente tomados por ações políticas do cotidiano, cultivando diversas possibilidades e ações relacionadas ao local e ao presente. Como fruto de processos de globalização e reflexividade, emergem novos valores universais e objetos de contestação e preocupação no campo dos movimentos sociais - como é o caso da alimentação, que se politiza sob o enfoque do ativismo alimentar.

$\mathrm{O}$ ativismo alimentar surge como um guarda-chuva interdisciplinar que abriga diferentes movimentos e discussões de amplo alcance nas sociedades, nos quais a comida aparece como elemento transversal, como a 
Agroecologia e a Agricultura Familiar; o movimento de Segurança Alimentar e Nutricional; a Agricultura Orgânica e outros sistemas agroalimentares sustentáveis (Permacultura, Agriculturas Biodinâmica, Natural, Ecológica); o Comércio Justo (Fair Trade); o Slow Food; o Locavorismo; o Vegetarianismo; o Veganismo; o Freeganismo, entre os mais conhecidos.

Sem ater-nos a cada um desses movimentos em profundidade, é importante destacar que diferentes e complexas reivindicações políticas estão por trás do que se identifica superficialmente como apelos a uma alimentação saudável e, por isso, muitos deles, não são compreendidos como movimentos sociais. A Agroecologia, por exemplo, não é um sistema produtivo sustentável que dispensa o uso de insumos sintéticos. Ela se utiliza de práticas de agricultura sustentável, mas se define como um movimento sociopolítico de fortalecimento do agricultor familiar, de resgate de suas identidades e raízes culturais e, principalmente, de sua autonomia, poder de decisão e participação ativa no processo produtivo, favorecendo o local como foco de ação.

A proposta de Segurança Alimentar e Nutricional, resultante da organização e influência da sociedade civil, não está preocupado somente em promover o acesso permanente e igualitário a uma alimentação saudável e adequada. O termo adequada abrange os aspectos do desenvolvimento social e econômico baseado em um modelo de produção de alimentos que expresse a soberania alimentar e que, ao mesmo tempo, estimule a revalorização de métodos tradicionais de manejo e gestão ambiental, baseados nos conhecimentos acumulados de populações locais em sua íntima convivência com o meio natural e a otimização dos recursos disponíveis nos distintos lugares para atender a necessidade de reprodução biológica e social dos seres humanos (Brasil, 2017a; 2010).

A Agricultura Orgânica e outras formas de sistemas agropecuários sustentáveis não estão interessadas somente em produzir alimentos sem 
agrotóxicos. Além de proibir o uso de muitos outros contaminantes como drogas veterinárias, fertilizantes, aditivos sintéticos e tecnologias como a transgenia e a irradiação, a Agricultura Orgânica, na legislação brasileira, é definida como um movimento que objetiva

a auto-sustentação da propriedade agrícola no tempo e no espaço, a maximização dos benefícios sociais para o agricultor, a minimização da dependência de energias não renováveis na produção, a oferta de produtos saudáveis que ponham em risco a saúde do consumidor, do agricultor e do meio ambiente, o respeito à integridade cultural dos agricultores e a preservação da saúde ambiental e humana (Brasil, 2007b).

Oliveira (2014) mostra em sua tese que o Slow Food se configura como um tipo de movimento social que, além de questionar a lógica da produção agroalimentar do sistema capitalista e suas repercussões sobre a saúde, o meio ambiente e a justiça social, também assume uma crítica estética e a valorização do prazer hedonista como diferencial de outras formas de ativismo.

Da mesma forma, as discussões que envolvem o Locavorismo (Azevedo, 2015), o Vegetarianismo e o Veganismo (Azevedo, 2013b) e o Freeganismo ${ }^{14}$ exigem fôlego para abarcar sua complexidade, que constrói relações com gênero, família e sexualidade; com a revitalização do meio rural; com os aspectos éticos na relação com o reino animal; com questões de mobilidade, consumo sustentável e preservação da agrobiodiversidade; com estratégias de vida em comunidade; com boicotes e alternativas ao sistema econômico neoliberal; com a promoção da cooperação e do decolonialismo cultural alimentar; com a exploração da natureza e de outros seres humanos, entre outras abordagens que obscurecem a relação

${ }^{14} \mathrm{O}$ Freeganismo é um movimento social e implica em práticas de coleta e aproveitamento de alimentos no espaço urbano. Mais informação no site que discute "Freeganism as a strategy for sustainable livings beyond capitalism": http://freegan.info/what-is-a-freegan/translations/o-que-e-freeganismo/ Acesso em 21 Mar 2015. 
imediata que remete a simples opção por dietas locais, a bases locais de origem vegetal para promover saúde ou resgatar práticas tradicionais.

Por fim, para além de uma perspectiva de criação de identidades coletivas que regem as decisões individuais e se opõem à lógica do sistema agroalimentar dominante, presente em quase todos tipos de ativismo alimentar, Ascher (2005) chama atenção para os significados coletivos dados aos alimentos através desses movimentos, que tendem a ocupar o lugar que a moral religiosa deixou vago, restaurando novas lógicas normativas através do comer. Essa, talvez, seja a chave para abrir o campo de estudos sobre alguns tipos de fundamentalismo alimentar inerentes a determinados estilos de vida. Cabe aqui uma referência ao estudo sobre neonazismo e vegetarianismo, que aborda a dieta vegetariana para além da expressão de um estilo de vida individual que carrega o estereótipo de posicionamento político de esquerda ou da contracultura (Tominic; Forchtner, 2017). Para os autores, em especial na Alemanha, a dieta isenta de carnes (bem como as preocupações ambientais) está historicamente relacionada ao espectro do espaço político de extrema direita, que conecta vegetarianismo a noções de pureza e superioridade racial.

Sob tais égides conceituais, novas qualidades adjetivam o já polissêmico conceito contemporâneo de alimento saudável na ótica dos ativistas: ecológico; orgânico; ético; local; alimento decolonial (e colonial); sustentável; tradicional; seguro e adequado; limpo; puro; alimento amigo do animal; alimento feito por mulheres; alimento afetivo; site specific food; comfort food... só para citar alguns que contribuem para fomentar a confusão dos comedores.

O ativismo alimentar ainda carece de estudos empíricos aprofundados que possam delimitar suas reais motivações, bem como conhecer as diferentes formas de ações utilizadas pelos ativistas, os atores envolvidos em cada instância política e sua abrangência e impacto na sociedade. O 
movimento tende a impulsionar o processo de politização do ato de se alimentar como resposta a práticas de violação do direito humano a uma alimentação de qualidade e a soberania e segurança alimentar.

\section{Considerações Finais}

Ao delinear os eixos temáticos desse estudo, observa-se que muitos temas dialogam, entrelaçam-se e permitem contaminações conceituais; ou seja, não são formas puras. É possível também afirmar que a alimentação vem ganhando destaque como objeto de análise social e as abordagens se multiplicam. A comida ganha notoriedade em diferentes formas de visualidade e mais legitimidade na arena das Ciências Humanas e Sociais. O amplo escopo teórico oferece diferentes olhares sobre os novos comedores, o(s) sistema(s) agroalimentar(es), sobre as formas de ação política e os novos movimentos sociais, sobre os estudos de gênero e da cultura contemporânea.

Analisando os estudos mais recentes, percebe-se que o cientista social interessado em alimentação deve cultivar um ânimo acadêmico para transitar em diferentes disciplinas e áreas de estudo. Os estudos interdisciplinares sobre alimentação tendem a dificultar a elaboração de molduras conceituais ou eixos temáticos como foi proposto nesse estudo. Essas relações interdisciplinares podem ser vantajosas para fortalecer a área da Sociologia da alimentação, mas podem também diluir o tema da comida enquanto subcampo de outras disciplinas e interferir na coesão intelectual da área.

Estudos futuros demandam legitimação empírica dentro dos diferentes eixos aqui propostos. Há carência de estudos que mostrem como os grupos se mobilizam frente às diferentes propostas de ativismo no país - um campo fértil para analisar fundamentalismo alimentar; veganismo 
e gênero; estudar a perspectiva política implícita no ato de se alimentar; explorar os conceitos de foodscape, alimento local e de locavorismo brasileiro. Seria importante saber como se comportam os consumidores e os diferentes atores (Estado, mídia, ciência, indústria alimentar) diante das questões éticas ligadas à alimentação e também frente aos riscos e controvérsias alimentares; o que move os foodies e a geração Yum brasileira - se é que eles existem por aqui; como é experiência social dos comedores que comem sozinhos nos centros urbanos. Muitas outras perguntas podem ser respondidas através dos estudos culturais da comida: quais alimentos são marcadores simbólicos da identidade do povo brasileiro? Diante da diversificação das estruturas de família, quem escolhe o que comer, prepara as refeições e por quê? Onde e por quem são os alimentos tradicionais preparados e qual o limite entre a autêntica herança culinária e as tradições inventadas? Como funcionam as dietas e novas práticas alimentares contemporâneas em termos de (des)agregação social? Qual o papel da alimentação na inserção social e na minimização do preconceito de imigrantes e refugiados transnacionais no Brasil contemporâneo?

Não se pretende aqui esgotar as questões ou as temáticas nos campos social e cultural da comida, mas mostrar a fertilidade e a variedade das pesquisas, bem como instigar novos estudos empíricas entre cientistas sociais, que cubram inevitáveis lacunas desse campo de conhecimento. Acredita-se que esse estudo deve contribuir para a construção de um estado da arte da pesquisa em alimentação, cultura e sociedade, evidenciando-a dentro de molduras mais porosas e instigantes inerentes às Ciências Sociais e Humanas.

Elaine de Azevedo é professora do Departamento de Ciências Sociais e Programa de Pós-Graduação de Ciências Sociais, Universidade Federal do Espírito Santo. $>$ elainepeled@gmail.com. 
Sociologias, Porto Alegre, ano 19, no 44, jan/abr 2017, p. 276-307

\section{Referências}

1. ADAMS, C.J. The Pornography of Meat. New York: Continuum International, 2003.

2. ADAMS, C.J. The Sexual Politics of Meat: A Feminist-Vegetarian Critical Theory. New York: Continuum International, 1990.

3. ALLAIN, J.M.; GUIVANT, J.S. O estudo da confiança na alimentação: análise do campo de pesquisa. Revista Brasileira de Informação Bibliográfica em Ciências Sociais - BIB, v.69, p. 79-96, 2010. Disponível em: http://www.anpocs.org/portal/ index.php?option $=$ com_docman\&task $=$ doc_download\&gid $=15 \&$ Itemid $=435$ Acesso em: 5 Mar 2015.

4. AlGRANTI, L.M.; ASFORA, W. Apresentação. Cadernos Pagu, n.39, pp. 7-13, 2012.

5. ARNOLD, D. Famine: social crisis and historical change (New Perspectives on the Past.). Oxford: Basil Blackwell, 1988.

6. ASCHER, F. Le mangeur hypermoderne: une figure de l'individu écletique. Paris: Odile Jacob, 2005.

7. ATKINS, P; BOWLER, I. Food in society, Economy, Culture, Geography. London: Arnold, 2001.

8. AZEVEDO, E. de.; PELED, S. Comida e Visualidades. Visualidades, v.14, n.2, pp. 30-47, 2016.

9. AZEVEDO, E. de.; PELED, Y. Artevismo Alimentar. Contemporânea. Revista de Sociologia da UFSCar, v.5, pp.495 520, 2015.

10. AZEVEDO, E. de. O Ativismo Alimentar na perspectiva do Locavorismo. Revista Ambientes e Sociedade, v.18, pp.81 98, 2015.

11. AZEVEDO, E. de. Segurança Alimentar e Nutricional e Controvérsias Científicas. Revista Segurança Alimentar e Nutricional, v.20, pp.147 - 155, 2013a.

12. AZEVEDO, E. de. Vegetarianismo. Demetra: Alimentação, Nutrição \& Saúde, v.8, pp.275 - 288, 2013 b.

13. AZEVEDO, E. de. Alimentos orgânicos: ampliando conceitos de saúde humana, social e ambiental. São Paulo: Ed SENAC, 2012.

14. AZEVEDO, E. de. Riscos e controvérsias na construção social do conceito de alimento saudável: o caso da soja. Revista de Saúde Pública, v.45, pp.781 - 788 , 2011.

15. BEARDSWORTH, A.; KEIL, T. Sociology on the menu. London: Routledge, 1997. 
16. BOURDIEU, P. Distinction: A Social Critique of the Judgement of Taste. Cambridge, MA: Harvard University Press, 1984.

17. BRANDÃO, C.R. Plantar, colher e comer. Rio de Janeiro: Graal, 1981.

18. BRASIL. Decreto no 7.272, de 25 de Agosto de 2010. Regulamenta a Lei no 11.346, que cria o Sistema Nacional de Segurança Alimentar e Nutricional. Diário Oficial [da] União, 26 ago 2010. Disponível em: http://www.planalto.gov. br/ccivil_03/_ato2007-2010/2010/decreto/d7272.htm Acesso em: 12 dez. 2012.

19. BRASIL. Conselho Nacional de Segurança Alimentar e Nutricional. CONSEA. Relatório Final. GT Alimentação Adequada e Saudável. 2007a. Disponível em: http://www.planalto.gov.br/Consea/static/documentos/Tema/AlimentacaoAdequa/ RelatorioFinal.pdf Acessado em: 15 mai. 2009.

20. BRASIL. Decreto № 6.323, de 27 de dezembro de 2007. Dispõe sobre a agricultura orgânicas e dá outras providências. Diário Oficial da União, Brasília, Seção 1, p.2, 28 dez, 2007 b.

21. CAROLAN, M. The Socioogy of Food and Agriculture. New York, London: Routledge, 2012.

22. CARVALHO, M.C.; LUZ, M. T. Simbolismo sobre"natural" na alimentação. Ciência \& Saúde Coletiva, v. 16, n. 1, pp. 147-154, 2011.

23. CASCUDO, L.C. História da Alimentação no Brasil. São Paulo: Global, 2004.

24. COLLAÇO, J. Imigração e cozinha italiana na cidade de São Paulo. Anuário Antropológico, 2013. Disponível em: http://aa.revues.org/327 Acesso em: 3 Mar 2016.

25. CONTRERAS, J.; GARCIA ANAÍZ, M. Alimentação, sociedade e cultura. Rio de Janeiro: Editora Fiocruz, 2011.

26. COUNIHAN, C.; VAN ESTERIK, P. Food and Culture. A Reader. United Kingdom, New York: Routledge, 2012.

27. COVENEY, J. Food, morals, and meaning: The pleasure and anxiety of eating. London: Routledge, 2006.

28. DAVID, M.L.; GUIVANT, J.S. A gordura trans: entre as controvérsias científicas e as estratégias da indústria alimentar. Política \& Sociedade, v. 11, pp. 49-74, 2012.

29. DÓRIA, C.A. O que é a gastronomia hoje. Dossiê Gastronomia é Cultura? Formação, Identidade e História da Alimentação. Revista Cult. São Paulo, n. 198, p. 19-23.18 fev. 2015.

30. DÓRIA, C.A. Formação da culinária brasileira. Três estrelas, 2014. 
31. DÓRIA, C.A. Flexionando o gênero: a subsunção do feminino no discurso moderno sobre o trabalho culinário. Cadernos Pagu, n.39, pp. 251-271, 2012.

32. FISCHLER, C. El (h)omnívoro. El gusto, la cocina y el cuerpo. Barcelona: Editorial Anagrama, 1995.

33. FISCHLER, C. Food, Self and Identity. Social Science Information, v. 27, pp. 275-293, 1988.

34. FLAMMANG, J.A. The taste for civilization: food, politics and civil society. Illinois: University of Illinois Press, 2009.

35. FLANDRIN, J.-L.; MONTANARI, M. História da Alimentação. São Paulo: Estação Liberdade, 1998.

36. FREITAS, M.do C.S. Agonia da fome. Rio de Janeiro: Editora FIOCRUZ; Salvador: EDUFBA, 2003.

37. FREYRE, G. Casa-Grande \& Senzala: formação da família brasileira sob o regime da economia patriarcal. Rio de Janeiro: José Olympio, 1977.

38. GASPARETTI, F. The cultural meaning of food and its polyvalent role in the construction of identity among Senegalese migrants in Italy. Food and Migration Workshop. 2009. School of Oriental and African Studies, University of London. SOAS. Disponível em: https://www.soas.ac.uk/migrationdiaspora/seminarsevents/food_migration_abstracts/file49144.pdf Acesso em: 20 Mar 2017.

39. GERMOV, J.; WILLIAMS, L. Sociology of Food and Nutrition: the social apetite. Oxford: Oxford University Press, 2008.

40. GIDDENS, A. As consequências da modernidade. São Paulo: Ed Unesp, 1991.

41. GREW, R. The Globalization of Food (review) Technology and Culture, v. 52, n.1, pp. 210-211, 2011.

42. GUIVANT, J. S. Transgênicos e percepção pública da ciência no Brasil. Ambiente \& Sociedade, v. 9, n. 1, 2006.

43. INGLIS, D.; GIMLIN, D. The Globalization of Food. Oxford, UK, New York: Berg Publishers, 2009.

44. LANG T.; BARLING D.; CARAHER M. Food Policy: integrating health, environment and society. Oxford: Oxford University Press, 2009.

45. LUHMANN, N. Trust and power: two works by Niklas Luhmann. Chichester: John Wiley \& Sons, 1979.

46. MACHADO, J.A. Ativismo em rede e conexões identitárias: novas perspectivas para os movimentos sociais. Sociologias, v.18, pp. 248-285, 2007. 
47. MACIEL, M.E. Identidade Cultural e Alimentação. In: CANESQUI, A.M.; GARCIA, R.W. (Org.). Antropologia e Nutrição: um diálogo possível. Rio de Janeiro: Editora da FIOCRUZ, pp. 49-55, 2005.

48. MARTIN, B. Activism, social and political. In: GARY, A.L.; G.L.; HERR, K. (Eds.) Encyclopedia of Activism and Social Justice. Thousand Oaks, CA: Sage, pp. 19-27, 2007.

49. MICHELETTI, M. Political Virtue and Shopping: Individuals, Consumerism, and Collective Action. New York: Palgrave, 2003.

50. MINTZ, S.W. Comida e antropologia: uma breve revisão. Rev. Bras. Ci. Soc., v.16, n.47, 2001.

51. MOSKOVICS, B.B. Restaurante em que só se come sem companhia questiona overdose social. Folha de São Paulo (online), 2015. Disponível em: http:// www1.folha.uol.com.br/comida/2015/08/1670173-restaurante-em-que-so-secome-sem-companhia-questiona-overdose-social.shtml Acesso em: 5 Dez 2015.

52. NASCIMENTO, A.B. Comida - prazeres, gozos e transgressões. Salvador: EDUFBA, 2007.

53. NESTLE, M. Safe Food. Berkley, Los Angeles, London. University of California Press, 2003.

54. NESTLE, M. Food Politics. Berkley, Los Angeles, London. University of California Press, 2002.

55. NORRIS, P. Political Activism: New Challenges, New Opportunities. In: BOIX, C.; STOKES, S. (Orgs). The Oxford Handbook of Comparative Politics. Oxford: Oxford University Press, pp. 628-649, 2007.

56. OLIVEIRA, D.C. de. Comida, carisma e prazer: um estudo sobre a constituição do Slow Food no Brasil. 2014. 210 fls. Tese (doutorado) Universidade Federal Rural do Rio de Janeiro, Instituto de Ciências Humanas e Sociais, 2014.

57. POLLAN, M. Em defesa da Comida. São Paulo: Intrínseca, 2008.

58. POLLAN, M. O dilema do Onívoro. São Paulo: Intrínseca, 2007.

59. PORTILHO, F.; CASTANEDA, M.; CASTRO, I. R.R. A alimentação no contexto contemporâneo: consumo, ação política e sustentabilidade. Ciência e Saúde Coletiva, Rio de Janeiro, v. 16, n. 1, Jan, 2011.

60. POULAIN, J. P. Dictionnaire des cultures alimentares. Paris: Presses Universitaires de France, 2012.

61. POULAIN, J. P. Sociologias da Alimentação. Florianópolis: Editora UFSC, 2004. 
62. PLUMWOOD, V. Integrating ethical frameworks for animals, humans, and nature: a critical feminist eco-socialist analysis. Ethics \& the Environment. v. 5, n. 2, pp. 285-322, 2000.

63. RIAL, C.S. Olhares antropológicos sobre a alimentação. Brasil: primeiros escritos sobre comida e identidade. In: CANESQUI, A.M.; GARCIA, R.W. (orgs). Antropologia e nutrição: um diálogo possível. Rio de Janeiro: Editora FIOCRUZ, pp.87-100, 2005.

64. RITZER, G. The McDonaldization of society. Thousand Oaks, London, New Deli: Pine Forge, 2004.

65. ROZIN, P. La préferénce pour le natural. In: FISCHLER, C.; MASSON, E. (eds). Manger. Français, Européens et Américans face à I'álimentation . Paris: Odile-Jacob, pp. 193-208, 2008.

66. ROZIN, P. Psychobiological and cultural determinants of food choice. Silverstone: Trevor, 1976.

67. RUDY, K. Locavores, Feminism, and the Question of Meat. The Journal of American Culture, v.35, pp. 26-36, 2012.

68. SANTILLI, J. Agrobiodiversidade e direitos dos agricultores. São Paulo: Petrópolis, 2009.

69. SANTOS, L.A.S. O corpo, o comer e a comida: um estudo sobre as práticas corporais alimentares cotidianas a partir da cidade de Salvador. Bahia. Salvador: EDUFBA, 2008.

70. SCHLOSSER, E. Fast Food Nation: the dark side of all American Meal. New York: Mariner Books, 2012.

71. SCHWADE E.; WOORTMANN, E.F. Entrevista: Prof. Klaas Woortmann (UnB). Vivência: Revista de Antropologia, v.1, n. 43, 2014.

72. SCRINIS, G. On the ideology of nutritionism. Gastronomica, v. 8, n.1, pp. 39-48, 2008.

73. SILIPRANDI, E. Políticas de alimentação e papéis de gênero: desafios para uma maior equidade. Caderno de Debates, v. XI, pp.38-57, 2004.

74. SILVA, N.C. Culinária e Alimentação em Gilberto Freyre: Raça, Identidade e Modernidade. Latin American Research Review, v.49, n.3, pp. 3-21, 2014.

75. SIMONSEN, R.R. A Queer Vegan Manifesto. Journal for Critical Animal Studies, v.10, n.3, pp. 51-80, 2012.

76. SINGER, P. Libertação animal. Porto Alegre: Lugano Editora, 2004. 
77. SPAARGAREN, G. Political consumerism for sustainable consumption practices. Rethinking the commitments of citizen-consumers with environmental change. In: SANTOS, M.; VOGT, C.; FRANÇA, J.G.; GUIVANT, J.(orgs); Ciência, Tecnologia e Sociedade. Novos Modelos de Governança. Brasília: CGEE, pp. 135-165, 2005.

78. TOMINC, A.; FORCHTNER, B. Kalashnikov and Cooking-spoon: Neo-nazism, Veganism and Lifestyle in Germany. Food, Culture and Society, v.20, n.3, 2017 [no prelo].

79. TRUNINGER, M. As bases plurais da confiança alimentar nos produtos orgânicos: da certificação ao 'teste da minhoca'. Ambiente \& Sociedade, v.16, n. 2, 2013 .

80. TUROW, E. Taste of Generation Yum: How the Millennial Generation's Love for Organic Fare, Celebrity Chefs, and Microbrews Will Make or Break the Future of Food. Kindle E-book, 2015.

81. VERTHEIN, U.; VÁZQUEZ-MEDINA, A. Os territórios do comer. Cult. Revista Brasileira de Cultura, São Paulo, ano 18, n. 198, pp. 26-29, fev. 2015.

82. WARD, P.; COVENEY, J.; HENDERSON, J. A sociology of food and eating: Why now? Journal of Sociology, v. 46, pp. 347-351, 2010.

83. WOOLLEY, K.; FISHBACH, A. A recipe for friendship: similar food consumption promotes trust and cooperation. Journal of Consumer Psychology, v. 27, n.1, pp.1-10, 2017.

84. WOORTMANN, K. O sentido simbólico das práticas alimentares. In: Coletânea de palestras do I Congresso de Gastronomia e Segurança Alimentar. Brasília: Universidade de Brasília; p.3. 2004.

85. WOORTMANN, K. A comida, a família e a construção do gênero feminino. Revista de Ciências Sociais. v. 29, n.1, 1986.

Recebido: 29.06.2015

Aceito: 05.01.2016 